\title{
Cytoskeleton disruption affects Kv2.1 channel function and its modulation by $\mathrm{PIP}_{2}$
}

\author{
Mayra Delgado-Ramírez ${ }^{1}$ Aldo A. Rodríguez-Menchaca ${ }^{1}$
}

Received: 1 November 2018 / Revised: 7 February 2019 / Accepted: 7 March 2019 / Published online: 21 March 2019

(c) The Physiological Society of Japan and Springer Japan KK, part of Springer Nature 2019

\begin{abstract}
Voltage-gated potassium channels are expressed in a wide variety of excitable and non-excitable cells and regulate numerous cellular functions. The activity of ion channels can be modulated by direct interaction or/and functional coupling with other proteins including auxiliary subunits, scaffold proteins and the cytoskeleton. Here, we evaluated the influence of the actinbased cytoskeleton on the Kv2.1 channel using pharmacological and electrophysiological methods. We found that disruption of the actin-based cytoskeleton by latrunculin B resulted in the regulation of the Kv2.1 inactivation mechanism; it shifted the voltage of half-maximal inactivation toward negative potentials by approximately $15 \mathrm{mV}$, accelerated the rate of closed-state inactivation, and delayed the recovery rate from inactivation. The actin cytoskeleton stabilizing agent phalloidin prevented the hyperpolarizing shift in the half-maximal inactivation potential when co-applied with latrunculin $\mathrm{B}$. Additionally, $\mathrm{PIP}_{2}$ depletion (a strategy that regulates Kv2.1 inactivation) after cytoskeleton disruption does not regulate further the inactivation of Kv2.1, which suggests that both factors could be regulating the Kv2.1 channel by a common mechanism. In summary, our results suggest a role for the actin-based cytoskeleton in regulating Kv2.1 channels.
\end{abstract}

Keywords $\mathrm{Kv}$ channels $\cdot$ Cytoskeleton $\cdot$ Latrunculin $\mathrm{B} \cdot$ Phalloidin $\cdot \mathrm{PIP}_{2} \cdot$ Patch clamp

\section{Introduction}

Voltage-gated potassium (Kv) channels control electrical excitability in multiple cell types. Change in membrane potential is the fundamental stimulus for Kv channel gating; however, other factors, such as protein phosphorylation $[1,2]$, SUMOylation [3, 4], intracellular $\mathrm{Ca}^{2+}[5]$, lipids [6, $7]$, accessory subunits $[8,9]$, and cytoskeletal proteins [10, 11] regulate this process.

The delayed rectifier Kv2.1 is expressed in a wide variety of excitable and non-excitable cells, and it is notable for its important role in regulating neuronal excitability [12], neuronal apoptosis $[13,14]$, and glucose-stimulated insulin secretion [15-17]. A feature of Kv2.1 is that it forms cellsurface clusters in hippocampal neurons [18], HEK293 cells [19], and pancreatic $\beta$-cells [20]. However, a population of

Aldo A. Rodríguez-Menchaca

aldo.rodriguez@uaslp.mx

1 Departamento de Fisiología y Biofísica, Facultad de Medicina, Universidad Autónoma de San Luis Potosí, Venustiano Carranza \#2405, Col. Los Filtros, 78210 San Luis Potosí, SLP, Mexico non-clustered channels is also present in the plasma membrane of these cells [21]. As Kv2.1 channels within the clusters are not conductive, whole-cell Kv2.1 currents are mostly derived from non-clustered channels [21]. Several factors regulate Kv2.1 cluster assembly and maintenance; for example, cluster formation requires an intact C-terminus in the channel [22]; the phosphorylation state of Kv2.1 also regulates clustering, as dephosphorylation of the channel promotes declustering [23]. The cytoskeleton also has an influence in Kv2.1 cluster formation and maintenance; clustered channels are corralled by a cytoskeleton-based perimeter fence, forming a stable cell surface structure [24]. Similarly, non-clustered Kv2.1 channels are modulated by phosphorylation [1], and other factors [7, 25]; however, the role of the cytoskeleton on the function of this population of channels has not been investigated in detail.

The cortical actin-based cytoskeleton consists of a lattice network of actin filaments that underlies and connects with the plasma membrane [26]. Cumulative evidence suggests that the actin cytoskeleton plays an important role in the regulation of $\mathrm{Kv}$ channels; for instance, cytoskeleton disruption upregulates the ionic and gating currents of Kv1.5 channels [10]; whereas an intact cytoskeleton is essential 
for Kv1.2 channel function [11]. Additionally, disruption of actin filaments induces a progressive increase in the rate of inactivation of $\mathrm{Kv} 3.3$ channels, with no effects on its activation mechanism [27]. Recently, we reported that inactivation curves of Kv2.1 channel recorded in inside-out patches are shifted $\sim 48 \mathrm{mV}$ to hyperpolarized potentials compared to those recorded in whole cells [7]. This effect was partially prevented by phosphatidylinositol 4,5-bisphosphate $\left(\mathrm{PIP}_{2}\right)$; however, additional factors presumably lost or damaged after patch excision, perhaps the cytoskeleton, could be involved in this difference.

In this study, we have examined the role of the cytoskeleton on the function of Kv2.1 channels. We found that cytoskeleton disruption regulates the inactivation mechanism of Kv2.1 channels, with no apparent effects on its activation. Moreover, $\mathrm{PIP}_{2}$ depletion after cytoskeleton disruption does not regulate further the inactivation of Kv2.1 channels, suggesting that both factors could be acting through a common mechanism.

\section{Materials and methods}

\section{Drugs}

Latrunculin B, phalloidin, and rapamycin (ready-made solution, $2.7 \mathrm{mM}$ ) were purchased from Sigma-Aldrich (St. Louis, MO, USA). Latrunculin B and phallodin were dissolved in dimethyl sulfoxide (DMSO) to get $10 \mathrm{mM}$ and $5 \mathrm{mM}$ stock solutions, respectively. The stock solutions were diluted to the final concentrations in the internal or external solution for the patch clamp recordings. The final concentration of DMSO in our experiments was $\leq 0.1 \%$, which did not modify the basal properties of the Kv2.1 channel.

\section{Cell culture and transfection}

Human embryonic kidney 293 [HEK-293] (ATCC@ CRL$1573^{\mathrm{TM}}$ ) cells were grown in $60-\mathrm{mm}$ tissue culture dishes (Corning, Corning, NY, USA) in Dulbecco's modified Eagle's medium (DMEM, GIBCO-Invitrogen, Grand Island, NY, USA) supplemented with $10 \%$ fetal bovine serum (Corning Life Sciences, Manassas, VA, USA) and 1\% Antibiotic Antimycotic solution (Sigma-Aldrich) in a humidifier incubator at $37^{\circ} \mathrm{C}\left(5 \% \mathrm{CO}_{2}\right)$. HEK-293 cells were transiently transfected with cDNAs encoding rKv2.1 subcloned in the pXoom vector (kindly provided by Dr. Diomedes Logothetis, Northeastern University, USA), LDR (Lyn11-targeted FRB)-CFP and FKBP (FK506-binding protein)-Inp54p (kindly provided by Dr. Bertil Hille, University of Washington, USA) with the use of Lipofectamine 2000 reagent (Invitrogen, Carlsbad, CA, USA) according to the manufacturer's instructions. For electrophysiological recordings, cells were used $24 \mathrm{~h}$ after transfection. As a marker for successfully transfected cells, cDNA encoding the enhanced green fluorescent protein (EGFP) was co-transfected with the cDNAs of interest.

\section{Electrophysiological recordings}

Macroscopic current recordings in HEK-293 cells were performed at room temperature $\left(22-24{ }^{\circ} \mathrm{C}\right)$ by using the whole-cell and inside-out configurations of the patch-clamp technique. Data acquisition and generation of voltage-clamp pulse protocols were carried out using an Axopatch 200B amplifier (Molecular Devices, Sunnyvale, CA, USA) and a Digidata 1440A interface (Molecular Devices) controlled by the pCLAMP 10 software (Molecular Devices). Micropipettes were pulled from borosilicate glass capillary tubes (World Precision Instruments, Sarasota, FL, USA) using a flaming/brown micropipette puller (Sutter Instruments, Novato, CA, USA). When micropipettes were filled with the pipette solution, tip resistance ranged from 1.5 to 2.5 $\mathrm{M} \Omega$. An agar- $\mathrm{KCl}$ bridge was used to ground the bath. For whole-cell recordings, the standard bath solution contained: $135 \mathrm{mM} \mathrm{NaCl}, 4 \mathrm{mM} \mathrm{KCl}, 1 \mathrm{mM} \mathrm{MgCl} 2,10 \mathrm{mM}$ HEPES, $1.8 \mathrm{mM} \mathrm{CaCl}_{2}$, and $10 \mathrm{mM}$ glucose (pH was adjusted to 7.4 with $\mathrm{NaOH}$ ). The pipette solution contained: $110 \mathrm{mM}$ $\mathrm{KCl}, 5 \mathrm{mM} \mathrm{MgCl}{ }_{2}, 5 \mathrm{mM} \mathrm{K}{ }_{4}$ BAPTA, and $5 \mathrm{mM} \mathrm{K}_{2} \mathrm{ATP}$ ( $\mathrm{pH}$ adjusted to 7.2 with $\mathrm{KOH}$ ). Inside-out patches were recorded by using the standard bath solution in the patch pipette, and the perfusing solution contained $135 \mathrm{mM} \mathrm{KCl}$, $1 \mathrm{mM} \mathrm{MgCl} 2,10 \mathrm{mM}$ HEPES, and $10 \mathrm{mM}$ glucose $(\mathrm{pH}$ adjusted to 7.35 with $\mathrm{KOH}$ ). Solutions were applied using a Fast-Step Perfusion System (VC-77SP Warner Instruments, Hamden, CT, USA).

\section{Data analysis}

Data are presented as mean \pm SEM ( $n=$ number of cells, recorded from at least four different experiments). Patchclamp data were processed using Clampfit 10 (Molecular Devices) and analyzed in Origin 8.6 (OriginLab Corp. Northampton, MA, USA).

Conductance-voltage $(G-V)$ relationships were determined based on the equation:

$G=\frac{I_{\mathrm{p}}}{V-V_{\text {rev }}}$,

where $I_{\mathrm{p}}$ is the peak current amplitude at the test potential $V$, and $V_{\text {rev }}$ is the potassium reversal potential. The voltage dependence of Kv2.1 channel activation was determined from the $\mathrm{G}-\mathrm{V}$ relationships fitted to a Boltzmann equation:

$y=\frac{1}{1+\mathrm{e}^{-\left(V-V_{1 / 2}\right) / K}}$, 
where $V$ represents the test potential, and $V_{1 / 2}$ and $K$ are the potential at which the conductance was half-activated and the slope, respectively.

The voltage dependence of Kv2.1 channel inactivation was determined using a three-step protocol. From a holding potential of $-100 \mathrm{mV}(-120 \mathrm{mV}$ for inside-out), a depolarizing $100 \mathrm{~ms}$ step to $+80 \mathrm{mV}$ was applied $\left(P_{1}\right)$; after a brief repolarization to the holding potential, a 6 -s conditioning pulse to different depolarizing potentials was applied $\left(P_{2}\right)$, followed by a final pulse to $+80 \mathrm{mV}\left(P_{3}\right)$. The normalized current was calculated dividing the current in $P_{3}$ between current in $P_{1}$ and plotted against the conditioning potential $\left(P_{2}\right)$. The resulting inactivation data were fitted with the Boltzmann equation:

$y=\frac{I_{\max }-I_{\min }}{1+\mathrm{e}^{\left(V-V_{1 / 2}\right) k}}+I_{\min }$

where $V$ is the conditional potential, and $V_{1 / 2}$ and $K$ are the potentials at which the conductance was half-inactivated and the slope, respectively.

The statistical significance between two or more independent experimental groups was determined by unpaired Student's $t$ test or one-way ANOVA respectively, the normality was determined by Shapiro-Wilk test, the homoscedasticity was evaluated by Brown Forsythe test, with $p<0.05$ representing significance.

\section{Results}

\section{Kv2.1 channels exhibit modulation of inactivation in inside-out patches}

We evaluated the voltage-dependence of inactivation of Kv2.1 channels recorded in the whole-cell and inside-out configurations of the patch-clamp technique. Representative
Kv2.1 current traces recorded with a three-pulse inactivation protocol (Fig. 1, inset) are shown for whole-cell (Fig. 1a) and inside-out (Fig. 1b) conditions. Half-maximal inactivation voltage $\left(V_{1 / 2}\right)$ and slope $(K)$ values determined from Boltzmann fits to inactivation curves are shown in Table 1. A gradual hyperpolarizing shift in the $V_{1 / 2}$ of inactivation was observed after patch excision (Fig. 1c). After $10 \mathrm{~min}$, the $V_{1 / 2}$ of inactivation was shifted $63 \mathrm{mV}$ to negative potentials compared to the $V_{1 / 2}$ obtained from whole-cell recordings (Fig. 1c, Table 1). The hyperpolarizing shift in the $V_{1 / 2}$ of Kv2.1 inactivation under inside-out conditions can be partially prevented by PIP $_{2}$ [7]; however, other factors could be contributing. In the next series of experiments, we evaluated if the cytoskeleton is involved in this effect.

\section{Effect of cytoskeleton disruption on Kv2.1 channels}

The influence of actin cytoskeleton on the function of ion channels can be investigated by disrupting actin polymerization. Latrunculin B (hereinafter Lat. B) is a marine toxin that inhibits actin polymerization and disrupts microfilament organization [28]. We investigated the effects of Lat. B on the activity of Kv2.1 channels. Whole-cell currents were elicited by depolarizing steps to $+60 \mathrm{mV}$ during $2 \mathrm{~s}$. Current

Table 1 Half-maximal inactivation voltage $\left(V_{1 / 2}\right)$ and slope $(K)$ of Kv2.1 channels under whole-cell and inside-out conditions

\begin{tabular}{llll}
\hline & $V_{1 / 2}(\mathrm{mV})$ & $K$ & $n$ \\
\hline Whole-cell & $-27.4 \pm 1.2$ & $5.6 \pm 0.3$ & 8 \\
Inside-out (1 min) & $-75.2 \pm 1.9^{* * *}$ & $4.8 \pm 0.1$ & 6 \\
Inside-out (5 min) & $-86.3 \pm 1.8^{* * *}$ & $6.1 \pm 0.23$ & 6 \\
Inside-out (10 min) & $-90.4 \pm 1.7^{* * *}$ & $6.2 \pm 0.18^{*}$ & 4 \\
\hline
\end{tabular}

$V_{1 / 2}$ and $k$ were calculated by fitting the Boltzmann equation (Eq. 3) to the inactivation curves in each cell/patch

${ }^{*} p<0.05, * * * p<0.001$ versus whole-cell (a)

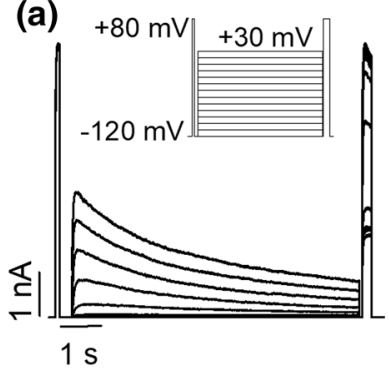

(b)

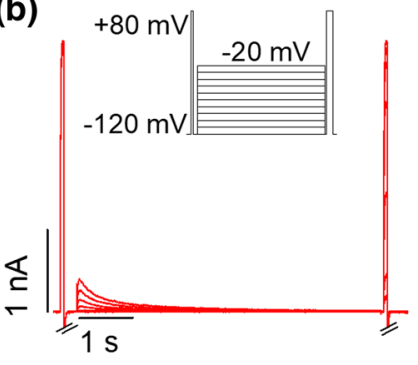

(c)

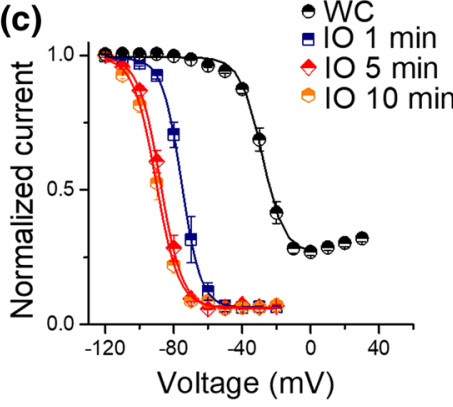

Fig. 1 Effects of patch excision on the voltage-dependent inactivation of Kv2.1 channels. a, b Representative recordings of Kv2.1 currents obtained with an inactivation protocol (inset) in whole-cell (a) and inside-out (b) configurations. c Inactivation curves of Kv2.1 channels determined under whole-cell (WC), and 1, 5, and 10 min after patch rupture (IO). Inactivation parameters are displayed on Table 1. Data are presented as mean \pm SEM 
traces obtained within 1 min of membrane rupture, and again after 25-min incubation with Lat. B in the patch pipette are shown in Fig. 2a. Lat. B $(5 \mu \mathrm{M})$ does not significantly affect the amplitude of Kv2.1 currents (Fig. 2b). For all subsequent experiments Lat. B $(5 \mu \mathrm{M})$ was dialyzed through the patch pipette during $25 \mathrm{~min}$ before starting the recordings. Similarly, untreated (control) cells were recorded after $25 \mathrm{~min}$ of the whole-cell configuration establishment.

To ascertain the effect of Lat. B on the voltage dependence of activation of Kv2.1 channels, we determined the conductance-voltage relationships $(G-V$ curves) from untreated (control) and Lat. B-treated cells. Figure 3a, b shows representative current traces recorded in untreated (control, a) and Lat. B-treated (b) cells, recorded with the protocol shown in the inset. Lat. B does not affect the voltage dependence of activation of Kv2.1 channels (Fig. 3c). The mean activation parameters in untreated and Lat. B-treated cells were $V_{1 / 2}=14.3 \pm 0.7 \mathrm{mV}, k=15.7 \pm 1.6 \mathrm{mV}$ and $V_{1 / 2}=8.3 \pm 2.5 \mathrm{mV}, k=21.2 \pm 1 \mathrm{mV}$, respectively.

Next, we determined the effect of Lat. B on the inactivation parameters of Kv2.1. Figure $4 a$, b shows representative current traces recorded in untreated (control, a) and Lat. B-treated (b) cells, recorded with the protocol shown in the inset. Lat. B induced a shift in the $V_{1 / 2}$ toward negative voltages relative to untreated cells (Fig. 4c). The $V_{1 / 2}$ in untreated cells was $-32.4 \pm 2.3 \mathrm{mV}(k=5.0 \pm 0.2 \mathrm{mV})$, while it was $-47.7 \pm 1.5 \mathrm{mV}(k=5.1 \pm 0.3 \mathrm{mV})$ in Lat. B-treated cells (i.e., a 15-mV leftward-shift, $p<0.001$ ).

A feature of Kv2.1 channels is that it can inactivate from closed states. We tested if Lab. B treatment can modulate the closed-state inactivation of Kv2.1. We isolated the closedstate inactivation of Kv2.1 by examining the development of inactivation at a non-activating voltage $(-40 \mathrm{mV})$, in untreated (control) and Lat. B-treated cells. In these experiments, a pulse to $+80 \mathrm{mV}$ tested the available current after the pre-pulse to $-40 \mathrm{mV}$; as the pre-pulse duration increased, channels inactivate and the current gradually

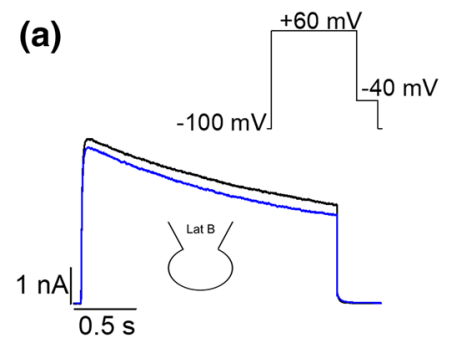

(b)

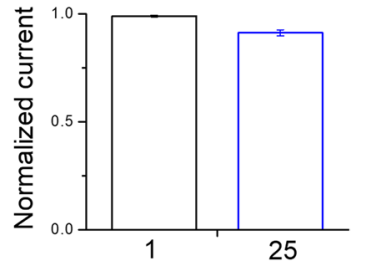

Fig. 2 Impact of cytoskeleton disruption on Kv2.1 currents. a Representative Kv2.1 currents recorded at $+60 \mathrm{mV}$ from a holding potential (hp) of $-80 \mathrm{mV}$ under whole-cell configuration. Traces are shown at 1 and $25 \mathrm{~min}$ after the establishment of the whole-cell configuration. Lat. B was dialyzed through the patch pipette. b Average current inhibition after 1 and 25 min of Lat. B dialysis $(n=6)$. Data are presented as mean \pm SEM
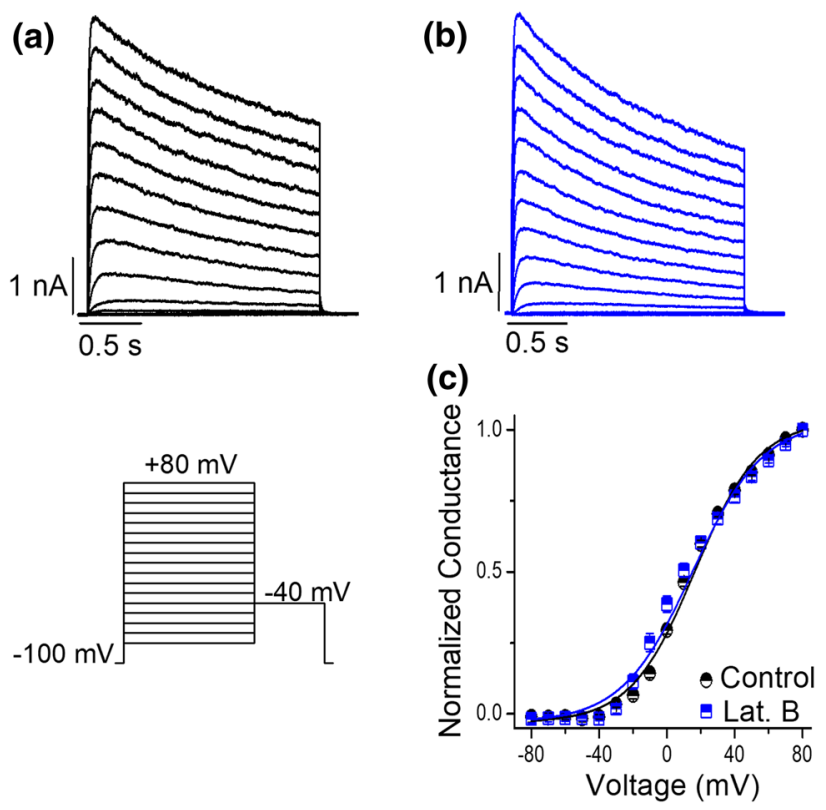

Fig. 3 Effect of cytoskeleton disruption on the voltage dependence of activation of Kv2.1 channels. a, b Representative recordings of Kv2.1 currents obtained in response to an activation protocol (inset) in untreated (control) and Lat. B-treated cells. c Activation curves of Kv2.1 channels determined in control conditions $(n=8)$ and Lat. B-treated cells $(n=6)$. Data are presented as mean \pm SEM
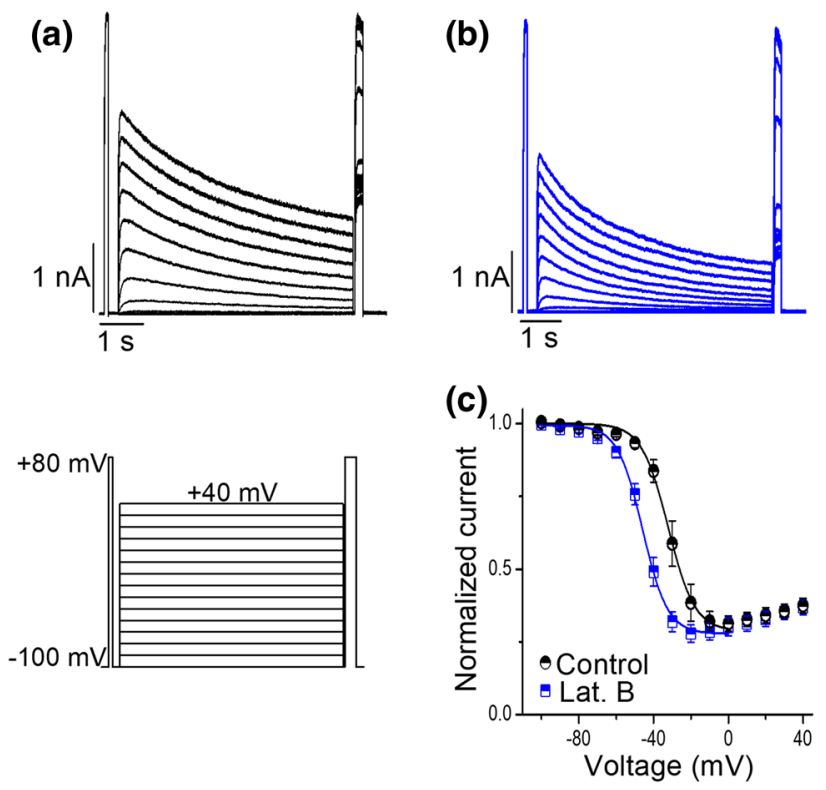

Fig. 4 Effect of cytoskeleton disruption on the voltage-dependence of inactivation of Kv2.1 channels. a, b Representative recordings of $\mathrm{Kv} 2.1$ currents obtained in response to an inactivation protocol (inset) in untreated (control) and Lat. B-treated cells. c Inactivation curves of Kv2.1 channels determined in control conditions $(n=6)$ and Lat. B-treated cells $(n=5)$. Data are presented as mean \pm SEM 
decreased. Figure 5a, b shows representative current traces recorded in untreated (control, a) and Lat. B-treated (b) cells, recorded with the protocol shown in the inset. The development of closed-state inactivation was accelerated in Lat. B-treated cells (Fig. 5c). The time constants of closedstate inactivation obtained from monoexponential fits were $16.5 \pm 1.6 \mathrm{~s}$ for control and $6.7 \pm 0.9 \mathrm{~s}$ for Lat. B-treated cells $(p<0.001)$.

To test if Lat. B treatment affected the recovery rate of Kv2.1 from inactivation, we used a three-pulse protocol (Fig. 6, inset). Figure 6a, b shows representative current traces obtained from untreated (control, a) and Lat. B-treated (b) cells. The recovery kinetics of Kv2.1 currents was fitted to a double exponential function that was affected by Lat. B (Fig. 6c). The recovery time constants were $0.21 \pm 0.02 \mathrm{~s}$ and $2.18 \pm 0.51 \mathrm{~s}$ for control, and $0.30 \pm 0.05 \mathrm{~s}$ and $2.57 \pm 0.43 \mathrm{~s}$ for Lat. B-treated cells $(p<0.05)$.

At this point, we tested if the effect on the voltage dependence of inactivation induced by Lat. B could be prevented by the F-actin stabilizing agent phalloidin. For these experiments, we had four experimental groups: untreated (control), Lat. B $(5 \mu \mathrm{M})$, phalloidin $(10 \mu \mathrm{M})$ and Lat. B $(5 \mu \mathrm{M})+$ phalloidin $(10 \mu \mathrm{M})$, all applied through the patch pipette. As previously shown, Lat. B induced a hyperpolarizing shift in the
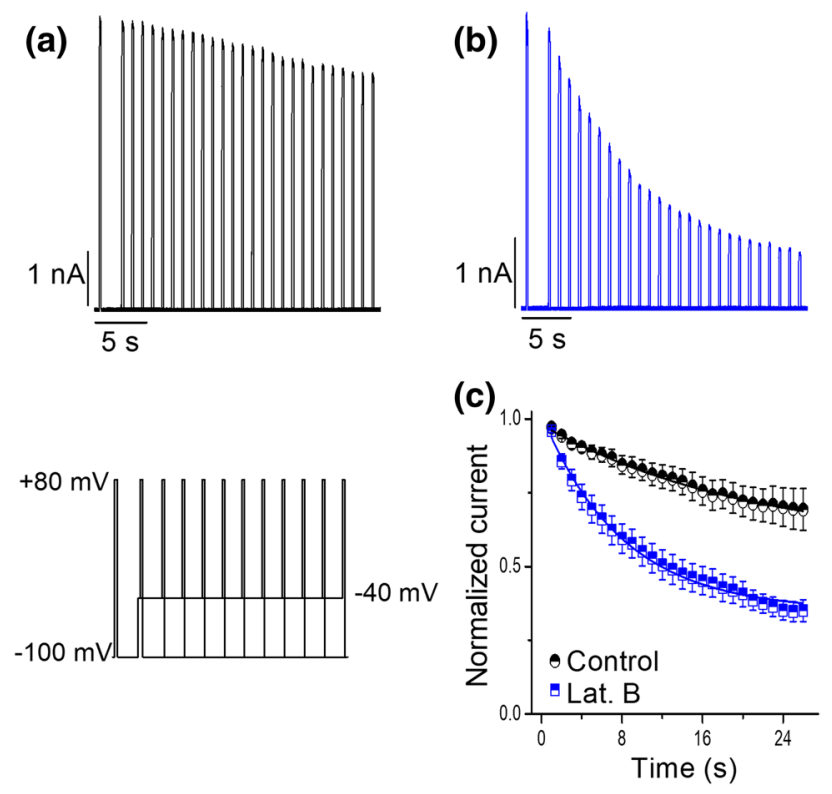

Fig. 5 Effect of cytoskeleton disruption on the kinetics of Kv2.1 closed-state inactivation. a, b Representative recordings of Kv2.1 currents obtained with a closed-state inactivation protocol (inset) in untreated (control) and Lat. B-treated cells. c Temporal course of closed-state inactivation determined in control conditions $(n=8)$ and Lat. B-treated cells $(n=7)$. The data points represent the current evoked by the second depolarizing pulse to $+80 \mathrm{mv}$, relative to the amplitude of the initial depolarizing pulse, plotted against the duration of the conditioning pulse at $-40 \mathrm{mV}$. Data are presented as mean \pm SEM
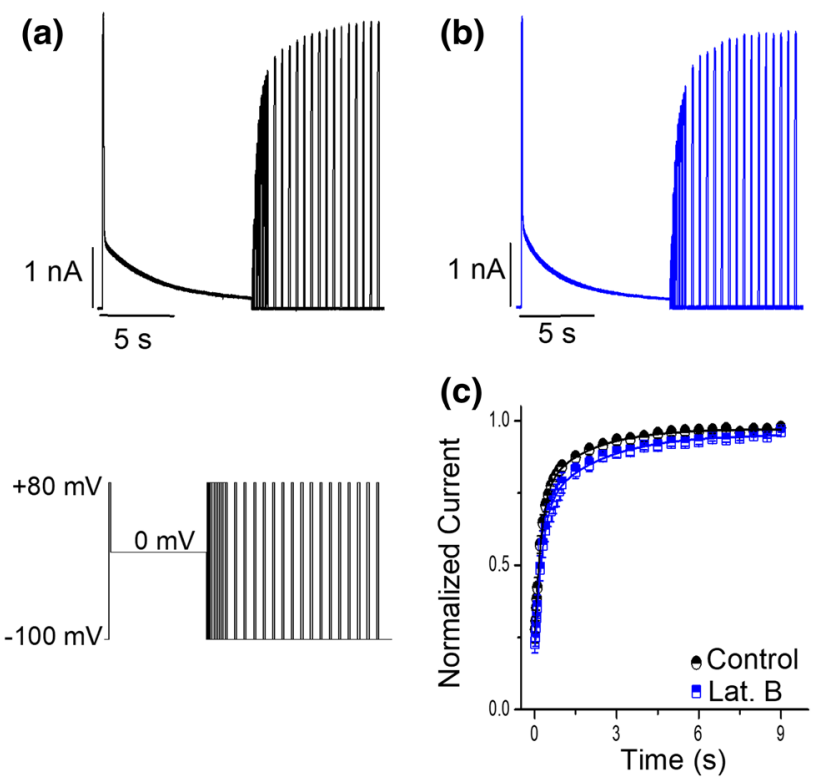

Fig. 6 Effect of cytoskeleton disruption on the recovery kinetics of Kv2.1 channels from inactivation. a, b Representative recordings of Kv2.1 currents obtained with a three-pulse protocol (inset) in untreated (control) and Lat. B-treated cells. c Temporal course of recovery from inactivation determined in control conditions $(n=5)$ and Lat. B-treated cells $(n=7)$. The data points represent the peak current evoked by the third pulse (P3), normalized to the peak current elicited by the identical first pulse (P1) and plotted as a function of the time interval at the holding potential $(-100 \mathrm{mV})$. Data are presented as mean $\pm \mathrm{SEM}$

$V_{1 / 2}$ of inactivation of $13.7 \mathrm{mV}$ (Fig. 7a, d). Phalloidin does not modify the voltage dependence of inactivation of Kv2.1 channels when it was applied alone (Fig. 7b, d); however, it was able to prevent the left-shift in the $V_{1 / 2}$ induced by Lat. B when they were applied together (Fig. $7 \mathrm{c}, \mathrm{d}) . V_{1 / 2}$ and $k$ values for the four experimental groups are shown in Table 2.

\section{$\mathrm{PIP}_{2}$ regulation of Kv2.1 channel depends on the cytoskeleton}

The effects on the inactivation mechanism of Kv2.1 channel induced by Lat. B are qualitatively and quantitatively similar to those induced by $\mathrm{PIP}_{2}$ depletion [7]. In experiments in excised patches, the $\mathrm{PIP}_{2}$ is dephosphorylated and the cytoskeleton destabilized, therefore, the combination of both factors could be inducing the large hyperpolarizing shift in the $V_{1 / 2}$ of inactivation observed in this recording configuration (Fig. 1c). Then, we examined the voltage dependence of inactivation of Kv2.1 channels after cytoskeleton disruption followed by $\mathrm{PIP}_{2}$ depletion. To deplete $\mathrm{PIP}_{2}$, we co-expressed Kv2.1 channels together with a rapamycin-translocatable phosphatase (FKBPInp54p) and the membrane anchor LDR-CFP. The FKBP and LDR domains dimerize when rapamycin is added to the cell, leading to the dephosphorylation of $\mathrm{PIP}_{2}$ on the 5' position to 
Fig. 7 Protective effect of phalloidin on Lat. B-induced shift on the voltage dependence of inactivation of Kv2.1 channels. a-c Inactivation curves of Kv2.1 channels determined in control conditions, Lat. B, phalloidin (Phall) and Lat. $\mathrm{B}+$ Phall-treated cells. For the sake of clarity, the control curve is shown compared to each different treatment. d Average $V_{1 / 2}$ value for control, Lat. B, Phall and Lat. B + Phall-treated cells (see Table 2). Data are presented as mean \pm SEM
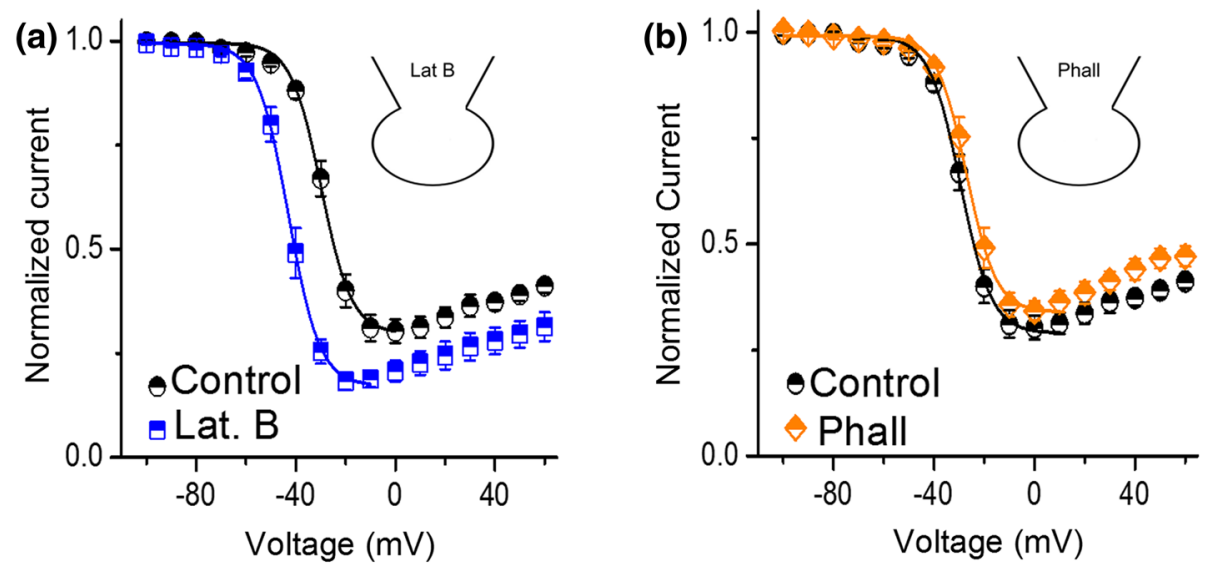

(c)

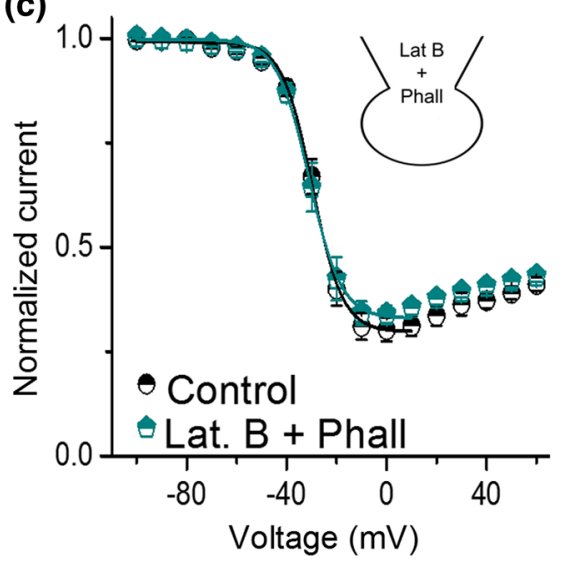

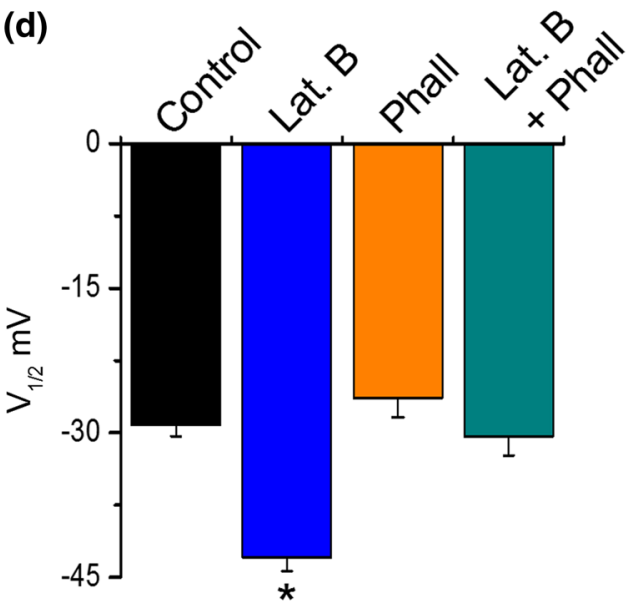

Table 2 Half-maximal inactivation voltage $\left(V_{1 / 2}\right)$ and slope $(K)$ of Kv2.1 channels in absence (control) and presence of Lat. B, phalloidin and Lat. B + phalloidin

\begin{tabular}{llll}
\hline & $V_{1 / 2}(\mathrm{mV})$ & $K$ & $n$ \\
\hline Control & $-29.2 \pm 1.2$ & $5.9 \pm 0.2$ & 6 \\
Lat. B & $-42.8 \pm 1.44^{* * *}$ & $5.6 \pm 0.2$ & 5 \\
Phalloidin & $-26.3 \pm 1.9$ & $5.8 \pm 0.4$ & 7 \\
Lat. B + phalloidin & $-30.4 \pm 1.9$ & $5.4 \pm 0.2$ & 7 \\
\hline
\end{tabular}

$V_{1 / 2}$ and $k$ were calculated by fitting the Boltzmann equation (Eq. 3) to the inactivation curves in each cell

*** $p<0.001$ versus control

PI4P [29]. Lat. B was dialyzed through the patch pipette during the experiment $(25 \mathrm{~min})$, whereas rapamycin $(1 \mu \mathrm{M})$ was added to the extracellular solution 15 min after establishing the whole-cell configuration. Under these conditions, the voltage dependence of inactivation was shifted to hyperpolarizing potentials by $15 \mathrm{mV}$ (Fig. 8c, f), which was not significantly different from the changes observed when Lat. B or rapamycin were applied alone (Fig. 8a, b, f). These experiments suggest that instead of having additive effects, as we expected, $\mathrm{PIP}_{2}$ depletion and the cytoskeleton disruption could be modulating
Kv2.1 channels by a common mechanism. To further explore this possibility, we first tested if $\mathrm{PIP}_{2}$ could prevent the hyperpolarizing shift on the voltage dependence of inactivation induced by Lat. B. PIP $2(10 \mu \mathrm{M})$ was co-applied with Lat. B trough the patch pipette $25 \mathrm{~min}$ before the recordings. Under this experimental setting, $\mathrm{PIP}_{2}$ was unable to circumvent the hyperpolarizing shift in the $V_{1 / 2}$ of inactivation induced by Lat. B (Fig. 8d, f). Additionally, we examined if the actin cytoskeleton stabilizing agent phalloidin could abolish the effects observed after $\mathrm{PIP}_{2}$ depletion. Phalloidin was dialyzed trough the patch pipette $15 \mathrm{~min}$ before the rapamycin application to deplete $\mathrm{PIP}_{2}$. Despite the cytoskeleton stabilization by phalloidin, PIP $_{2}$ depletion induced a hyperpolarizing shift in the voltage dependence of inactivation of Kv2.1 (Fig. 8e, f). The $V_{1 / 2}$ and $k$ values for the six experimental groups are shown in Table 3.

\section{Discussion}

The Kv2.1 channel is unique among voltage-gated potassium channels in that it is present in the plasma membrane concentrated in clusters or as a single (non-clustered) 
(a)

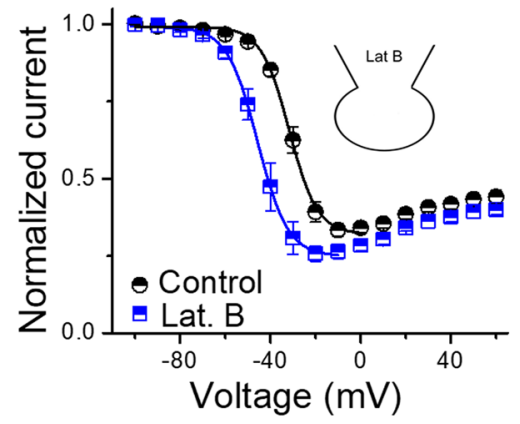

(d)

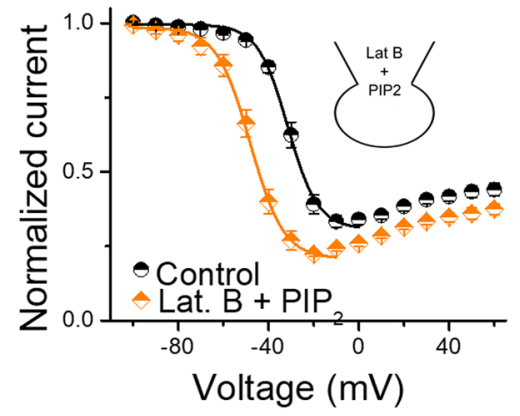

(b)

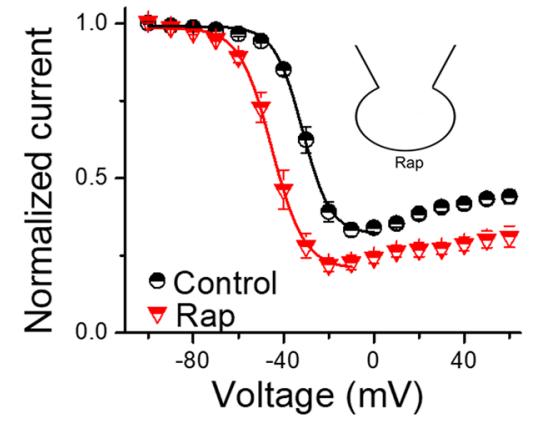

(e)

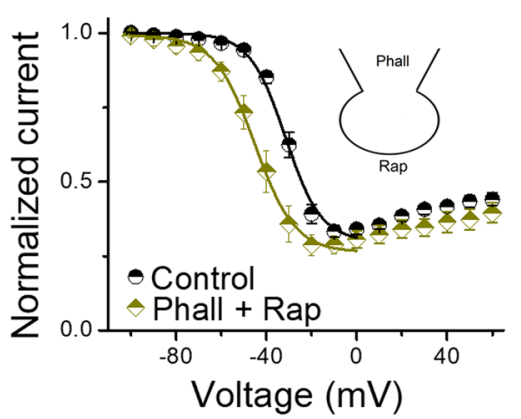

(c)
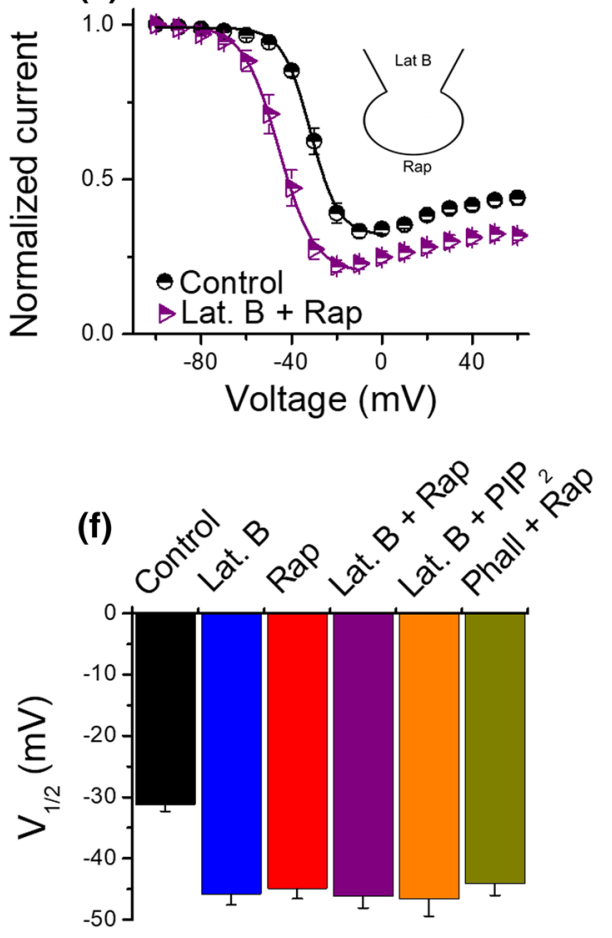

Fig. 8 Effect of the cytoskeleton and $\mathrm{PIP}_{2}$ on the voltage dependence of inactivation of Kv2.1 channels. a-e Inactivation curves of Kv2.1 channels determined in control conditions, Lat. B, rapamycin (Rap), Lat. B + Rap, Lat. B + PIP 2 and Phall + Rap-treated cells. For the sake

Table 3 Half-maximal inactivation voltage $\left(V_{1 / 2}\right)$ and slope $(K)$ of $\mathrm{Kv} 2.1$ channels in absence (control) and presence of Lat. B, rapamycin, Lat. B + rapamycin, Lat. B + $\mathrm{PIP}_{2}$ and phalloidin + rapamycin

\begin{tabular}{llll}
\hline & $V_{1 / 2}(\mathrm{mV})$ & $K$ & $n$ \\
\hline Control & $-31.2 \pm 1.1$ & $5.7 \pm 0.5$ & 8 \\
Lat. B & $-45.8 \pm 1.8^{* * *}$ & $5.9 \pm 0.3$ & 6 \\
Rapamycin & $-44.9 \pm 1.7^{* * *}$ & $6.4 \pm 0.1^{*}$ & 7 \\
Lat. B + rapamycin & $-46.2 \pm 1.9^{* * *}$ & $5.4 \pm 0.1$ & 10 \\
Lat. B + PIP 2 & $-46.6 \pm 2.8^{* * *}$ & $5.4 \pm 0.2$ & 5 \\
Phalloidin + rapamycin & $-44.1 \pm 1.9^{* * *}$ & $8.8 \pm 0.8^{* *}$ & 6 \\
\hline
\end{tabular}

$V_{1 / 2}$ and $k$ were calculated by fitting the Boltzmann equation (Eq. 3) to the inactivation curves in each cell

${ }^{*} p<0.05, * * p<0.01, * * * p<0.001$ versus Control

freely diffusing channel [18-21]. Clustered channels do not conduct potassium; therefore, the non-clustered channels are responsible for the delayed rectifier currents attributed to Kv2.1 [21]. The role of the actin cytoskeleton in the regulation of Kv2.1 channel clustering has been studied in detail [24, 30, 31]. However, the regulation of non-clustered Kv2.1 channels by the actin cytoskeleton remains less explored. Here, we have investigated the role of the actin cytoskeleton on conductive (non-clustered) of clarity, the control curve is shown compared to each different treatment. $f$, average $V_{1 / 2}$ value for control, Lat. B, Rap, Lat. B + Rap, Lat. $\mathrm{B}+\mathrm{PIP}_{2}$ and phall + Rap-treated cells (see Table 3). Data are presented as mean \pm SEM

channels by employing the actin-disrupting agent Lat B. We found that cytoskeleton disruption regulates the inactivation mechanism of the Kv2.1 channel and its modulation by $\mathrm{PIP}_{2}$.

Several Kv channels interact with the actin cytoskeleton. As an example, Kv3.3 channels coordinate an assembly of cortical actin networks that in turn interact with channels and regulates its inactivation. The proposed mechanism is that cytoskeletal structures interact with the $\mathrm{N}$-terminus of Kv3.3 and interfere with the access of the inactivation ball to the channel pore [27]. Our results also suggest that the Kv2.1 channel is regulated by the cytoskeleton. Depolymerization of the actin cytoskeleton by Lat. B profoundly and exclusively affected the inactivation gating of Kv2.1 channels. The voltage of half-maximal inactivation is shifted toward negative potentials (Fig. 4), the rate of closed-state inactivation is accelerated (Fig. 5), and the recovery rate from Kv2.1 inactivation is delayed (Fig. 6). We did not find changes in the voltage dependence of activation, in agreement with previously reported results [21]. Phalloidin, an actin cytoskeleton stabilizing agent, prevented the inactivation changes induced by Lat. B (Fig. 7), supporting our interpretation that the effect of Lat. B is specific and mediated through depolymerization of actin filaments. 
In a previous work, we found that $\mathrm{PIP}_{2}$ regulates the inactivation gating of Kv2.1 channels [7]. Our findings here after disrupting the actin filaments are qualitatively and quantitatively similar to those induced by $\mathrm{PIP}_{2}$ depletion. We originally thought that both factors could combine to explain the large differences in the inactivation between whole-cell and inside-out experiments; however, our results suggest that Kv2.1 channels are regulated by the cytoskeleton and $\mathrm{PIP}_{2}$ by a non-additive and presumably common mechanism (Fig. 8). Moreover, cytoskeleton and $\mathrm{PIP}_{2}$ are both required for the regulation of $\mathrm{Kv} 2.1$ inactivation, as none of them can prevent the effects induced by the lack of the other (Fig. 8d-f), supporting a common mechanism of regulation.

It is well known that $\mathrm{PIP}_{2}$ binds to, and influences the activity of, many cytoskeletal proteins [32]. The cytoskeletal proteins regulated by $\mathrm{PIP}_{2}$ can in turn bind to other proteins and regulate its activity, as is the case of the NMDA receptor [33]. The C-terminal domain of NMDA receptors binds $\alpha$-actinin, which in turns binds to $\mathrm{PIP}_{2}$ in the plasma membrane; in this way, $\mathrm{PIP}_{2}$ and $\alpha$-actinin together regulate the opening of NMDA receptor [33]. A similar scenario could be responsible for the regulation of Kv2.1 channels, however, additional experiments are needed to corroborate this hypothesis. Currently, Kv2.1 has been shown to directly interact with proteins of the exocytic machinery [34, 35] and proteins of the endoplasmic reticulum [36, 37]; however, there are no reports of direct interactions between Kv2.1 and actin or actin binding proteins.

Another plausible explanation is that both cytoskeleton disruption and $\mathrm{PIP}_{2}$ depletion regulate $\mathrm{Kv} 2.1$ channels by inducing changes in the mechanical properties of the plasma membrane, as both strategies have been shown to alter the lipid bilayer properties [38, 39].

In summary, our data indicate that non-clustered Kv2.1 channels are also regulated by the actin-based cytoskeleton, which could have an influence on the excitability of Kv2.1expressing cells.

Acknowledgements We thank Xóchitl Ordaz Ruiz for technical assistance.

Author contributions M.D-R.: performed experiments, contributed to discussion, wrote/reviewed/edited manuscript. A.A.R-M.: conceived the project and contributed to discussion, wrote/reviewed/edited manuscript.

Funding This work was supported by SEP-CONACYT grants CB-157245 and CB-284443 (to A.A.R-M.) and CONACYT-FDC 2016-01-1995. M.D-R was supported by a Student Fellowship from CONACYT, México (374053).

\section{Compliance with ethical standards}

Conflict of interest The authors declare that there are no conflicts of interest.
Ethical approval This article does not contain any studies with human participants or animals performed by any of the authors.

\section{References}

1. Mohapatra DP, Park KS, Trimmer JS (2007) Dynamic regulation of the voltage-gated Kv2.1 potassium channel by multisite phosphorylation. Biochem Soc Trans 35:1064-1068. https://doi. org/10.1042/bst0351064

2. Cerda O, Trimmer JS (2010) Analysis and functional implications of phosphorylation of neuronal voltage-gated potassium channels. Neurosci Lett 486:60-67. https://doi.org/10.1016/j.neule t.2010.06.064

3. Dai XQ, Kolic J, Marchi P, Sipione S, Macdonald PE (2009) SUMOylation regulates Kv2.1 and modulates pancreatic betacell excitability. J Cell Sci 122:775-779. https://doi.org/10.1242/ jcs.036632

4. Plant LD, Dowdell EJ, Dementieva IS, Marks JD, Goldstein SA (2011) SUMO modification of cell surface Kv2.1 potassium channels regulates the activity of rat hippocampal neurons. J Gen Physiol 137:441-454. https://doi.org/10.1085/jgp.201110604

5. Gamper N, Li Y, Shapiro MS (2005) Structural requirements for differential sensitivity of KCNQ K+ channels to modulation by Ca2+/calmodulin. Mol Biol Cell 16:3538-3551. https://doi. org/10.1091/mbc.E04-09-0849

6. Rodriguez-Menchaca AA, Adney SK, Tang QY, Meng XY, Rosenhouse-Dantsker A, Cui M, Logothetis DE (2012) PIP2 controls voltage-sensor movement and pore opening of $\mathrm{Kv}$ channels through the S4-S5 linker. Proc Natl Acad Sci USA 109:E2399_ 2408. https://doi.org/10.1073/pnas.1207901109

7. Delgado-Ramirez M, De Jesus-Perez JJ, Arechiga-Figueroa IA, Arreola J, Adney SK, Villalba-Galea CA, Logothetis DE, Rodriguez-Menchaca AA (2018) Regulation of Kv2.1 channel inactivation by phosphatidylinositol 4,5-bisphosphate. Sci Rep 8:1769. https://doi.org/10.1038/s41598-018-20280-w

8. Pongs O, Leicher T, Berger M, Roeper J, Bahring R, Wray D, Giese KP, Silva AJ, Storm JF (1999) Functional and molecular aspects of voltage-gated $\mathrm{K}+$ channel beta subunits. Ann N Y Acad Sci 868:344-355

9. Aimond F, Kwak SP, Rhodes KJ, Nerbonne JM (2005) Accessory Kvbeta1 subunits differentially modulate the functional expression of voltage-gated $\mathrm{K}+$ channels in mouse ventricular myocytes. Circ Res 96:451-458. https://doi.org/10.1161/01.res.0000156890 .25876 .63

10. Maruoka ND, Steele DF, Au BP, Dan P, Zhang X, Moore ED, Fedida D (2000) Alpha-actinin-2 couples to cardiac Kv1.5 channels, regulating current density and channel localization in HEK cells. FEBS Lett 473:188-194

11. Williams MR, Markey JC, Doczi MA, Morielli AD (2007) An essential role for cortactin in the modulation of the potassium channel Kv1.2. Proc Natl Acad Sci USA 104:17412-17417. https ://doi.org/10.1073/pnas.0703865104

12. Mohapatra DP, Misonou H, Pan SJ, Held JE, Surmeier DJ, Trimmer JS (2009) Regulation of intrinsic excitability in hippocampal neurons by activity-dependent modulation of the KV2.1 potassium channel. Channels (Austin, Tex) 3:46-56

13. Pal S, Hartnett KA, Nerbonne JM, Levitan ES, Aizenman E (2003) Mediation of neuronal apoptosis by Kv2.1-encoded potassium channels. J Neurosci 23:4798-4802

14. Yao H, Zhou K, Yan D, Li M, Wang Y (2009) The Kv2.1 channels mediate neuronal apoptosis induced by excitotoxicity. J Neurochem 108:909-919. https://doi.org/10.111 $1 /$ j.1471-4159.2008.05834.x 
15. Jacobson DA, Kuznetsov A, Lopez JP, Kash S, Ammala CE, Philipson LH (2007) Kv2.1 ablation alters glucose-induced islet electrical activity, enhancing insulin secretion. Cell Metab 6:229235. https://doi.org/10.1016/j.cmet.2007.07.010

16. Li XN, Herrington J, Petrov A, Ge L, Eiermann G, Xiong Y, Jensen MV, Hohmeier HE, Newgard CB, Garcia ML, Wagner M, Zhang BB, Thornberry NA, Howard AD, Kaczorowski GJ, Zhou YP (2013) The role of voltage-gated potassium channels Kv2.1 and Kv2.2 in the regulation of insulin and somatostatin release from pancreatic islets. J Pharmacol Exp Ther 344:407-416. https ://doi.org/10.1124/jpet.112.199083

17. Tamarina NA, Kuznetsov A, Fridlyand LE, Philipson LH (2005) Delayed-rectifier (KV2.1) regulation of pancreatic beta-cell calcium responses to glucose: inhibitor specificity and modeling. Am J Physiol Endocrinol Metab 289:E578-585. https://doi. org/10.1152/ajpendo.00054.2005

18. Lim ST, Antonucci DE, Scannevin RH, Trimmer JS (2000) A novel targeting signal for proximal clustering of the Kv2.1 K+ channel in hippocampal neurons. Neuron 25:385-397

19. O'Connell KM, Tamkun MM (2005) Targeting of voltage-gated potassium channel isoforms to distinct cell surface microdomains. J Cell Sci 118:2155-2166. https://doi.org/10.1242/jcs.02348

20. Fu J, Dai X, Plummer G, Suzuki K, Bautista A, Githaka JM, Senior L, Jensen M, Greitzer-Antes D, Manning Fox JE, Gaisano HY, Newgard CB, Touret N, MacDonald PE (2017) Kv2.1 clustering contributes to insulin exocytosis and rescues human beta-cell dysfunction. Diabetes 66:1890-1900. https://doi.org/10.2337/ db16-1170

21. O'Connell KM, Loftus R, Tamkun MM (2010) Localizationdependent activity of the Kv2.1 delayed-rectifier K+ channel. Proc Natl Acad Sci USA 107:12351-12356. https://doi. org/10.1073/pnas.1003028107

22. Mohapatra DP, Trimmer JS (2006) The Kv2.1 C terminus can autonomously transfer Kv2.1-like phosphorylation-dependent localization, voltage-dependent gating, and muscarinic modulation to diverse Kv channels. J Neurosci 26:685-695. https://doi. org/10.1523/jneurosci.4620-05.2006

23. Misonou H, Mohapatra DP, Park EW, Leung V, Zhen D, Misonou $\mathrm{K}$, Anderson AE, Trimmer JS (2004) Regulation of ion channel localization and phosphorylation by neuronal activity. Nat Neurosci 7:711-718. https://doi.org/10.1038/nn1260

24. Tamkun MM, O'Connell KM, Rolig AS (2007) A cytoskeletalbased perimeter fence selectively corrals a sub-population of cell surface Kv2.1 channels. J Cell Sci 120:2413-2423. https://doi. org/10.1242/jcs.007351

25. Delgado-Ramirez M, Moran-Zendejas R, Arechiga-Figueroa IA, Toro-Castillo C, Ramirez-Martinez JF, Rodriguez-Menchaca AA (2016) Modulation of the voltage-gated potassium channel Kv2.1 by the anti-tumor alkylphospholipid perifosine. Pharmacol Rep 68:457-461. https://doi.org/10.1016/j.pharep.2015.11.006

26. Svitkina TM (2018) Ultrastructure of the actin cytoskeleton. Curr Opin Cell Biol 54:1-8. https://doi.org/10.1016/j.ceb.2018.02.007

27. Zhang Y, Zhang XF, Fleming MR, Amiri A, El-Hassar L, Surguchev AA, Hyland C, Jenkins DP, Desai R, Brown MR, Gazula VR, Waters MF, Large CH, Horvath TL, Navaratnam D, Vaccarino FM, Forscher P, Kaczmarek LK (2016) Kv3.3 channels bind Hax-1 and Arp2/3 to assemble a stable local actin network that regulates channel gating. Cell 165:434-448. https://doi. org/10.1016/j.cell.2016.02.009

28. Spector I, Shochet NR, Blasberger D, Kashman Y (1989) Latrunculins-novel marine macrolides that disrupt microfilament organization and affect cell growth: I. Comparison with cytochalasin D. Cell Motil Cytoskeleton 13:127-144. https://doi. org/10.1002/cm.970130302

29. Suh BC, Inoue T, Meyer T, Hille B (2006) Rapid chemically induced changes of PtdIns(4,5)P2 gate KCNQ ion channels. Science 314:1454-1457. https://doi.org/10.1126/science.1131163

30. Scannevin RH, Murakoshi H, Rhodes KJ, Trimmer JS (1996) Identification of a cytoplasmic domain important in the polarized expression and clustering of the Kv2.1 K+ channel. J Cell Biol 135:1619-1632

31. Weigel AV, Tamkun MM, Krapf D (2010) Anomalous diffusion of kv2.1 channels observed by single molecule tracking in live cells. Conf Proc IEEE Eng Med Biol Soc 2010:3005-3008. https://doi. org/10.1109/IEMBS.2010.5626150

32. Sechi AS, Wehland J (2000) The actin cytoskeleton and plasma membrane connection: PtdIns $(4,5) \mathrm{P}(2)$ influences cytoskeletal protein activity at the plasma membrane. J Cell Sci $113(\mathrm{Pt}$ 21):3685-3695

33. Michailidis IE, Helton TD, Petrou VI, Mirshahi T, Ehlers MD, Logothetis DE (2007) Phosphatidylinositol-4,5-bisphosphate regulates NMDA receptor activity through alpha-actinin. J Neurosci 27:5523-5532. https://doi.org/10.1523/JNEUR OSCI.4378-06.2007

34. MacDonald PE, Wang G, Tsuk S, Dodo C, Kang Y, Tang L, Wheeler MB, Cattral MS, Lakey JR, Salapatek AM, Lotan I, Gaisano HY (2002) Synaptosome-associated protein of 25 kilodaltons modulates Kv2.1 voltage-dependent $\mathrm{K}(+)$ channels in neuroendocrine islet beta-cells through an interaction with the channel $\mathrm{N}$ terminus. Mol Endocrinol 16:2452-2461. https://doi.org/10.1210/ me.2002-0058

35. Leung YM, Kang Y, Gao X, Xia F, Xie H, Sheu L, Tsuk S, Lotan I, Tsushima RG, Gaisano HY (2003) Syntaxin 1A binds to the cytoplasmic $\mathrm{C}$ terminus of $\mathrm{Kv} 2.1$ to regulate channel gating and trafficking. J Biol Chem 278:17532-17538. https://doi. org/10.1074/jbc.M213088200

36. Kirmiz M, Vierra NC, Palacio S, Trimmer JS (2018) Identification of VAPA and VAPB as Kv2 channel-interacting proteins defining endoplasmic reticulum-plasma membrane junctions in mammalian brain neurons. J Neurosci 38:7562-7584. https://doi. org/10.1523/JNEUROSCI.0893-18.2018

37. Johnson B, Leek AN, Sole L, Maverick EE, Levine TP, Tamkun MM (2018) Kv2 potassium channels form endoplasmic reticulum/plasma membrane junctions via interaction with VAPA and VAPB. Proc Natl Acad Sci USA 115:E7331-E7340. https://doi. org/10.1073/pnas.1805757115

38. Rusinova R, Hobart EA, Koeppe RE 2nd, Andersen OS (2013) Phosphoinositides alter lipid bilayer properties. J Gen Physiol 141:673-690. https://doi.org/10.1085/jgp.201310960

39. Martinac B (2014) The ion channels to cytoskeleton connection as potential mechanism of mechanosensitivity. Biochim Biophys Acta 1838:682-691. https://doi.org/10.1016/j.bbame m.2013.07.015

Publisher's Note Springer Nature remains neutral with regard to jurisdictional claims in published maps and institutional affiliations. 